\title{
A Novel Approach of Task Scheduling for Cloud Computing using Adaptive Firefly
}

\author{
Jasmeen Kaur \\ P.G. Student, \\ Department of Computer Engineering, \\ Sri Guru Granth Sahib World University, \\ Fatehgarh sahib, Punjab, India
}

\author{
Vinay Bhardwaj \\ Associate Professor, \\ Department of Computer Engineering, \\ Sri Guru Granth Sahib World University, \\ Fatehgarh sahib, Punjab, India
}

\begin{abstract}
Cloud Computing organization deals with large scale, large amount of data, and the demand of computing power, needs to increase system investment. It is one of efficient technology that is popular now days in IT field. The paper proposes an Adaptive firefly algorithm for solving the job scheduling problem in cloud computing. The results of the algorithm were tested on cloudsim- 3.0 by varying the configuration of virtual machines. After running the algorithm for different sets of jobs given to cloudsim-3.0, it is concluded that the results of Adaptive firefly are quite better than ACO.
\end{abstract}

\section{Keywords}

ACO, AFA, Task Scheduling.

\section{INTRODUCTION}

Cloud Computing is a model for conveying and hosting services on the internet. This model played important role when anybody started any organization from low level and add more resources according to the need. It is new computing technology that aims to provide quality of service, reliable for user. It is an extension of Distributed computing, Grid computing and parallel computing. It defined by the US National institute of standards and Technology states that cloud computing is a model for on demand network access to shared computing resource. It is paradigm for distributed computing that delivers infrastructure, software as services and platform. With the increase in the number of systems in the world it became necessary to provide those systems to process faster jobs with the same configuration. To achieve such complexities, computation should be performed somewhere on other system with high configuration, for that technology is shifting toward cloud. Cloud Computing organization deals with large scale, large amount of data, and the demand of computing power, need to increase system investment. It is one of efficient technology that is popular now days in IT field. Many change in the computing industry due to the cloud computing. It helps user applications provider of dynamic services using large scalable, secure, quick, data storage and virtualized resources over internet.

Its advantages only get when it is connect to the internet that's why user can use the powerfully computing services. It provides services according to user requirement. Virtualization provides technical support for cloud computing applications and virtualization technology. It is shared large number of services and resources provides for multiple users. Cloud model has three delivery model and four deployment models because of its operational and economic benefits. It shared resource and depends on the economics of scale, same as power network. It changed the client server. It provides many facilities not only the end-user but also the enterprise and organizations. It sorts architecture in particular public, private and hybrid so on. Cloud computing capacity planning dynamic upload and download the resources accordingly. It achieves the minimum waiting time, maximum throughput and good performance.

The main aim of job scheduling to distribute the system load, improve system performance, proper utilization the available resource to reduce the cost and execution time. It is play important role in cloud computing. It reduce the energy consumption require a minimum power. Jobs are schedule by user need. New scheduling strategies require to be proposed to overcome the problems posed by network properties between user and resource. The main advantages of scheduling algorithm obtain a high performance and proper utilization of resources. Job scheduling are the key technologies of cloud computing plays a vital role in an efficient use resource Management.

\subsection{TYPES OF SERVICES}

Cloud Computing architecture has three layers for the software which require on demand services over internet. Figure1. Shows different layer cloud architecture.

\subsubsection{Infrastructure as a service (IaaS)}

It is a first layer of Service. This layer delivers software and hardware components as a services. Sometimes IaaS is called a hosted service. Its capability provided to the user of IaaS as raw storage space, network resources and computing that's why user can run and execute applications, any software that they choose. Example of IaaS includes Flexi scale, Go grid, Amazon EC2.

\subsubsection{Platform as service (PaaS)}

It is a second layer of service. This layer uses the developer's application. It provider not only provides hardware, but also provide a toolkit and number of supported to higher level language. This layer use cloud application, Automatic Scaling, Load balancing with other services (For example Google Documents) are the major benefits to cloud application.

\subsubsection{Software as a Service (SaaS)}

This layer hosts the software and provides service to the customer through Internet. It reduces the maintenance cost of the customer. It is based on pay -as-go model. Cloud computing release their application on a hosting environment that can be access through networks by application user. Examples of SaaS include Google mail, Google docs and so forth. 


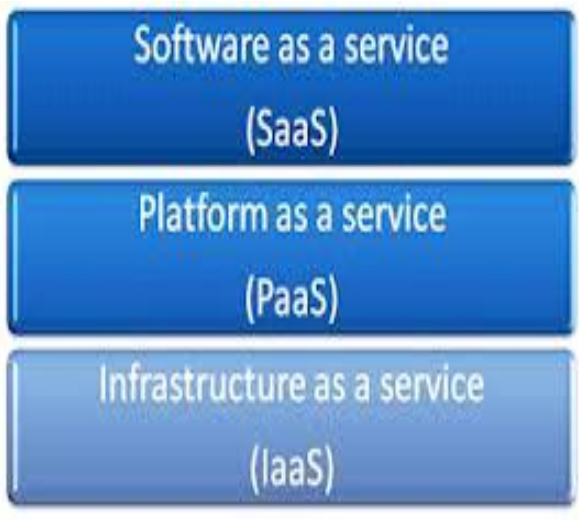

Fig. 1: Types of services

\section{RELATED WORKS}

Bilgaiyan S. et al. (2015) presented job scheduling is one of the important factor that affect the cloud performance and resource utilization. This paper discusses the some best swarm based scheduling techniques. These techniques compare on the bases of parameter, application and advantages. Every technique has its own parameters and objective, some based on minimize total execution time. Other is based on maximization throughput [1].

Naik P. et al. (2015) discuss the various job scheduling algorithms. The paper also includes proposed system with improved quality of service. A proposed system is based on bipartite matching. In which resource are allocated on different cloud overload condition and under-load is proposed system. The user are requested for jobs and executed, based on certain parameter that are already define for user request, it includes the storage space required for each job. Cloud environment include virtual machine to perform effective allocation [4].

Tawfeek M. et al. (2015) proposed Task Scheduling based on Ant Colony Optimization. One of the major issues in work is related to task scheduling. Simulation results demonstrate that ACO algorithm performs FCFS and RR algorithms.[5]

Jing et al. (2013) This paper is based on the Multi objective Genetic algorithm. It include the encoding rules like selection operators, crossover operators, method the sorting Pareto solutions. It is implementation the open source cloud computing simulation platform cloud-sim and compared with the existing technique. The result of the proposed system is better solution. It provides balance for the performance of multiple objectives [6]

Dillon T. et al. (2010) proposed all the challenges and issues that is face by the cloud computing. It is fast developing technology. It is increase day by day, now many company adopted the cloud computing environment, because it decrease the hardware cost and software cost. But all of these advantages even face the some challenging and issues. In this paper highlight issues and discussed [7]

Suraj P.et al. (2010) define the Particle swarm optimization technique .Particle swarm optimization is based on heuristics scheduling application. It is based on the data transmission cost and computational cost. The objective of this paper is minimizing the total execution time and minimizes total cost execution of application. The total cost is varying on the communication and transmission cost. Compare the particle swarm optimization result with 'Best Resource selection' heuristic. The result shows the particle swarm optimizations achieves better compare to BRS [8].

\section{PROPOSED METHODOLOGY}

In the cloud computing the advance facility provided by the service provided SaaS, PaaS and IaaS is security and optimal resource utilization so that every user gets the benefits of the services. Although services achieved by providing better algorithm in each area of concern are good yet sometimes they fail to meet real time outcomes. Therefore it becomes necessary to schedule and prioritize jobs. In Job Scheduling is divided into two categories i) Static scheduling ii) Dynamic scheduling, in which many techniques about job scheduling has been studied and few drawbacks in existing techniques were recognized. Those drawbacks motivated to have improvement in existing techniques and provide better solution to the user.

The paper proposes an Adaptive firefly algorithm for solving the job scheduling problem in cloud computing. The approach has two aspects. The First approach deals with the development of the cloud framework for job scheduling while the second with the development of firefly algorithm which would be applied on the cloud so as to improve the job scheduling scheme. The number of resources should be timely processed "used by each cloud user based" on the time occupied and network access charges. The Job scheduling algorithm will not only focus on whether the total time required to complete the task is minimized, but also on the time cost for finishing the subtasks. This will prevent the misallocation of time and resources in the multiple tasks performed by the cloud users. Figure below represents the flow of proposed methodology.

The developed algorithm will be applied on the cloud computing environment and the jobs will be scheduled according to the algorithm. The algorithms performance will be evaluated and monitored. The proposed technique will be implemented in Cloud Sim toolkit and the results are compared to those used in earlier literature in terms of execution time.

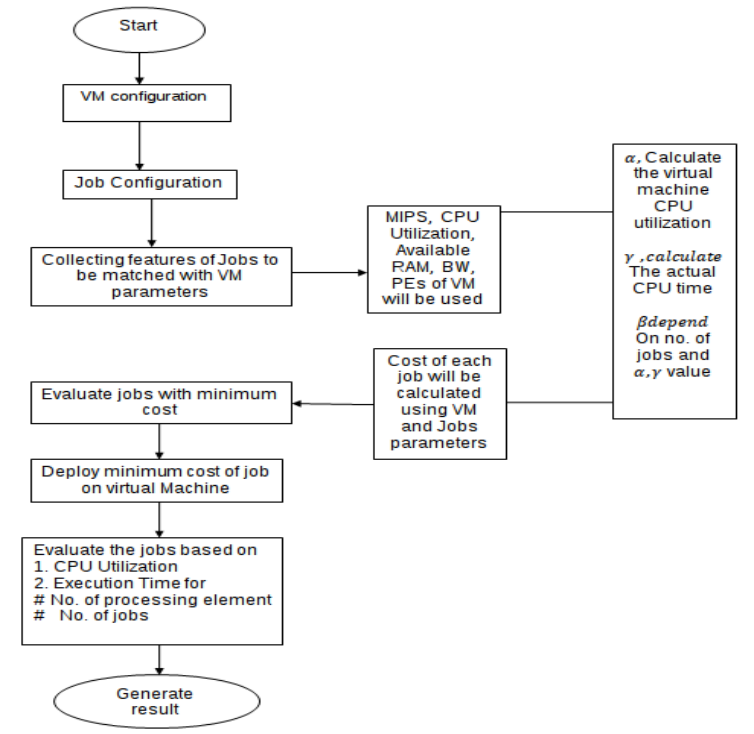

Fig 2: Flow chart of proposed methodology

\section{RESULTS}

Calculate Value of the ACO and Adaptive Firefly. This value is calculated based on jobs parameters. First of all discuss the job parameters. Job parameter include the Number of jobs, Number of Processing Element, Length of jobs /Total job size 
(MB), File size which is to be Processed, Output size /size of each job.

Formula for calculate length of jobs $=$ Number of job $*$ File size of job

Here the comparison among the two different optimization algorithms is done on the basis of their execution time which means the overall time taken by the cloud server to run or execute all the jobs provided to it. The comparison table of both the algorithm on different simulation environment depending on the number of jobs and processing elements is shown in table 1 .

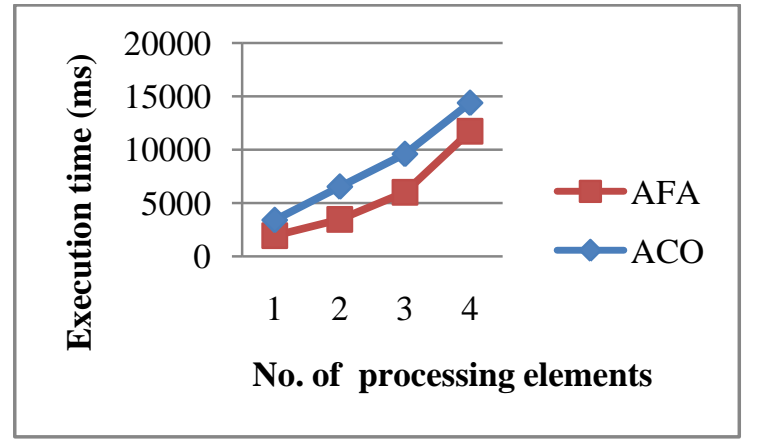

Fig 3: AFA v/s ACO keeping no. of jobs fixed at 100 and varying no. of Processing Elements

Graphs are made between Adaptive Firefly Algorithm (AFA) with Ant Colony Optimization (ACO). Fig.3shows the total execution time taken by the AFA and ACO for different no. of processing elements required by the jobs keeping number of jobs fixed at 100 to execute over the virtual machines.

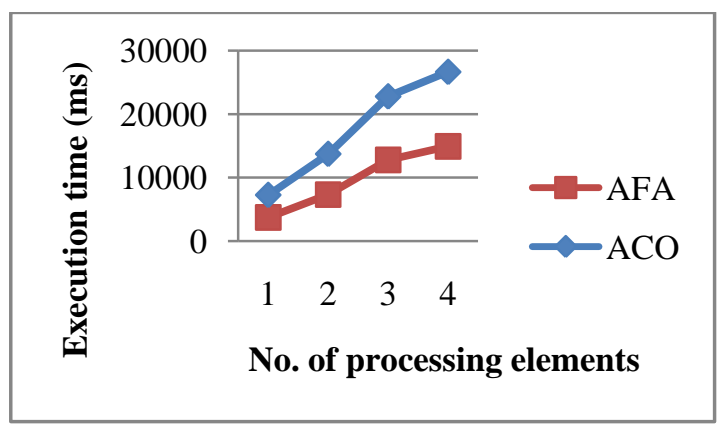

Fig 4: AFA v/s ACO keeping no. of jobs fixed at 200 and varying no. of Processing Elements
Graphs are prepared between AFA with ACO. Fig.4 shows the total execution time taken by the AFA and ACO for different no. of processing elements required by the jobs keeping number of jobs fixed at 200 to execute over the virtual machines.

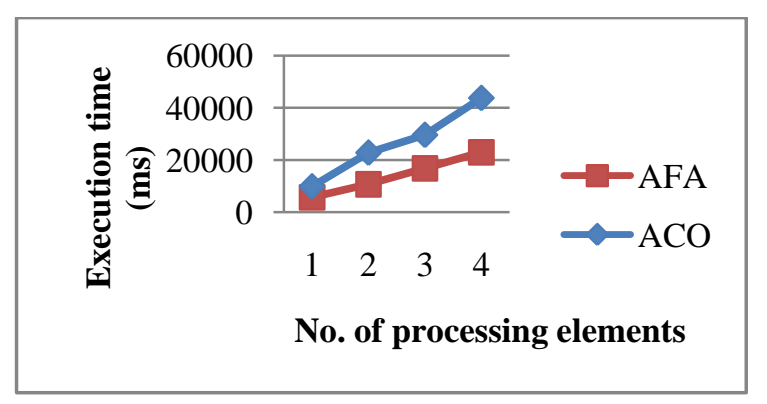

Fig 5: AFA v/s ACO keeping no. of jobs fixed at 300 and varying no. of Processing Elements

This graph compared the ACO and AFA. Fig.5it shows the total execution time taken by the AFA and ACO for different no. of processing elements required by the jobs keeping number of jobs fixed at 300 .

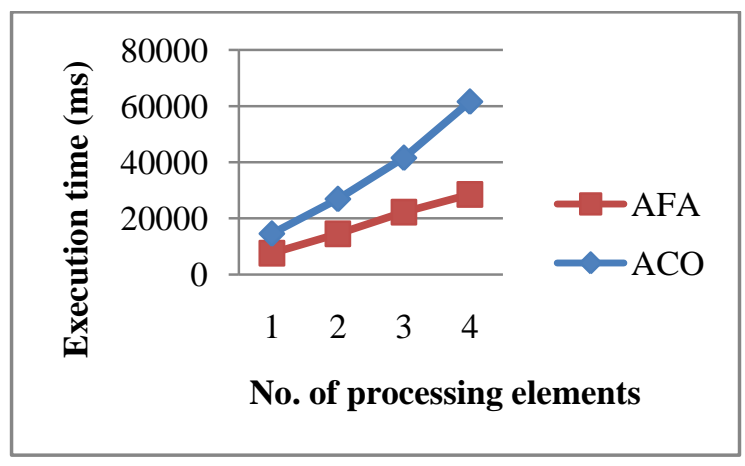

Fig 6: AFA v/s ACO keeping no. of jobs fixed at 400 and varying no. of Processing Elements

Figure 6 show the comparison graph between Adaptive Firefly Algorithm (AFA) with Ant Colony Optimization (ACO). It shows the total execution time taken by the AFA

Table 1: Execution time with varying number of jobs and processing element

\begin{tabular}{|c|c|c|c|}
\hline No. of Jobs & No. of PE's & ACO(Execution time) & AFA(Execution time) \\
\hline $\mathbf{1 0 0}$ & 1 & 3400.02 & 3733.33 \\
\hline $\mathbf{2 0 0}$ & 1 & 7266.59 & 5666.66 \\
\hline $\mathbf{3 0 0}$ & 1 & 9866.51 & 7599.93 \\
\hline $\mathbf{4 0 0}$ & 1 & 14599.74 & 3466.73 \\
\hline $\mathbf{1 0 0}$ & 2 & 6533.36 & 7333.37 \\
\hline $\mathbf{2 0 0}$ & 2 & 13733.26 & 10666.64 \\
\hline $\mathbf{3 0 0}$ & 2 & 22799.82 & \\
\hline
\end{tabular}




\begin{tabular}{|c|c|c|c|}
\hline 400 & 2 & 26933.1 & 14533.26 \\
\hline 100 & 3 & 9600.05 & 6000.1 \\
\hline 200 & 3 & 22799.92 & 12800.02 \\
\hline 300 & 3 & 29599.86 & 16799.98 \\
\hline 400 & 3 & 41599.78 & 22199.96 \\
\hline 100 & 4 & 14400.01 & 11733.37 \\
\hline 200 & 4 & 26666.69 & 14933.35 \\
\hline 300 & 4 & 43733.17 & 22933.31 \\
\hline 400 & 4 & 61599.72 & 28533.26 \\
\hline \multicolumn{4}{|c|}{ VM Configuration \{ Size: 5000 MB, RAM: 512, Mips: 200, BandWidth: 1500, } \\
\hline
\end{tabular}

and $\mathrm{ACO}$ for different no. of processing elements required by the jobs keeping number of jobs fixed 400 .

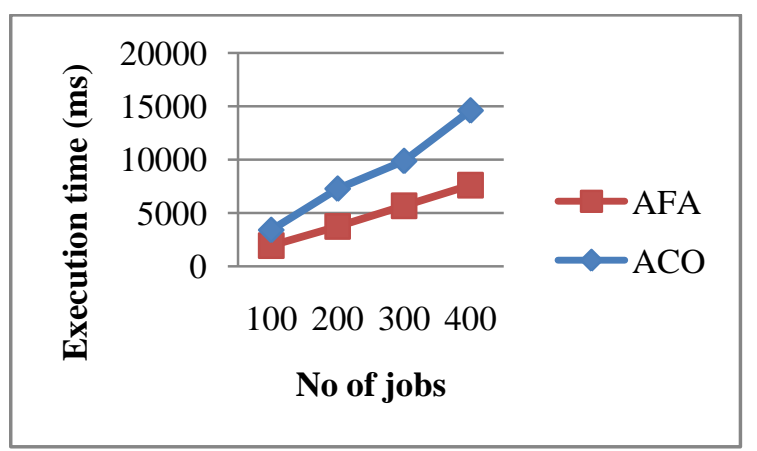

Fig 7: AFA v/s ACO keeping no. of processing elements fixed at 1 and varying no. of jobs

Figure 7 compared the graph of AFA and ACO. It shows the time taken by both the approach by varying the number of jobs executing over the virtual machine keeping the number of processing element fixed at 1 .

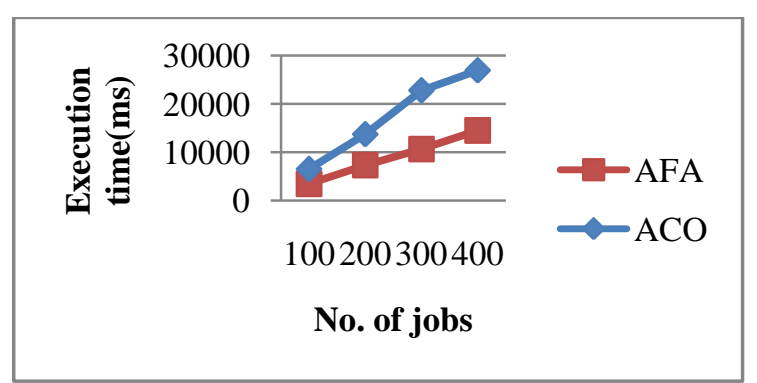

Fig 8: AFA v/s ACO keeping no. of processing elements fixed at 2 and varying no. of jobs

Figure 8 shows the difference between the graph of AFA and ACO. It shows the time taken by both the approach by varying the number of jobs executing over the virtual machine keeping the number of processing element fixed at 2 .

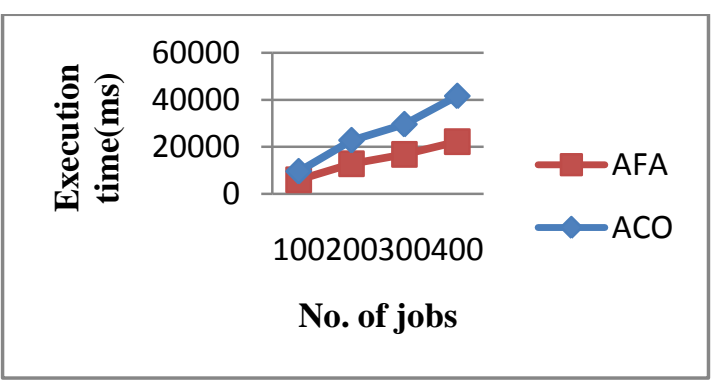

Fig 9: AFA v/s ACO keeping no. of processing elements fixed at 3and varying no. of jobs

Graphs are made between AFA and ACO. Fig. 9 it shows the time taken by both the approach by varying the number of jobs executing over the virtual machine keeping the number of processing element fixed at 2 .

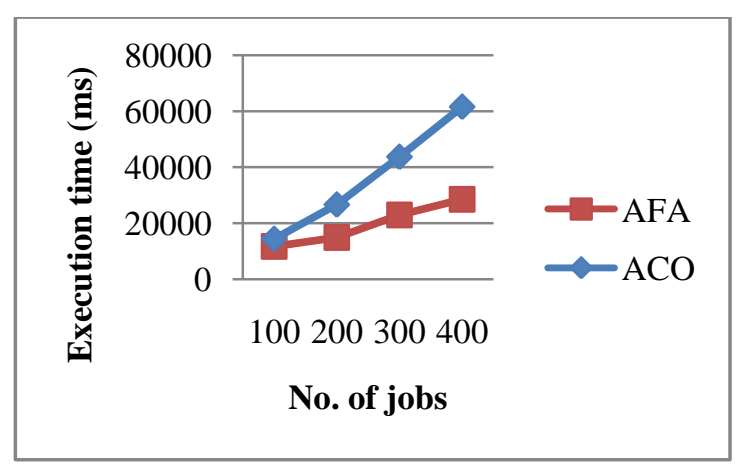

Fig 10: AFA v/s ACO keeping no. of processing elements fixed at 4 and varying no. of jobs

Figure 10 shows the comparison graph of AFA and ACO. It shows the time taken by both the approach by varying the number of jobs executing over the virtual machine keeping the number of processing element fixed at 4 . It is been cleared the time taken by AFA is less than that by ACO. 


\section{CONCLUSION AND FUTURE SCOPE}

Scheduling is one of the most important tasks in the cloud computing environment. In this research work algorithm for Ant Colony Optimization and Adaptive firefly were coded. The results of the algorithm were tested on cloudsim-3.0 by varying the configuration of virtual machines. After running the algorithm for different sets of jobs given to cloudsim-3.0, it is concluded that the results of Adaptive firefly are quite better than ACO. As the total time taken by the Adaptive firefly is less as compared to the earlier one, hence the number of jobs send over the cloud will execute faster as compared to other system. So, by adding the new parameter $\alpha$ to the algorithm of firefly, taking extra virtual machines configuration and cloudlets/jobs status parameters to modify the equation of $\alpha, \beta, \& \gamma$ and hence Intensity of the firefly to make it Adaptive firefly, to achieve the better results.

With the increasing scope of Cloud Computing in today's market. It has become important for developer/managers to schedule their job more efficiently. This research work emphasized on prioritizing high intensity jobs. In proposed work all the virtual machines deal with all kind of jobs. So our future works include the Setting up of virtual machine for specific task (which may be that of user or system) for the high performance. Our work can be extended by using different simulators like Workflow-sim.

\section{REFERENCES}

[1] Bilgaiyan, Saurabh, et al. "Study of Task Scheduling in Cloud Computing Environment Using Soft Computing Algorithms". International Journal of Modern Education and Computer Science (IJMECS) 7.3 (2015): 32.
[2] Buyya, Rajkumar, Rajiv Ranjan, and Rodrigo N. Calheiros. "Modeling and simulation of scalable Cloud computing environments and the CloudSim toolkit: Challenges and opportunities." High Performance Computing \& Simulation,2009. HPCS'09 International Conference on IEEE, 2009.

[3] Chang, Fangzhe, Jennifer Ren, and Ramesh Viswanathan. "Optimal resource allocation in clouds." Cloud Computing (CLOUD), 2010 IEEE 3rd International Conference on IEEE, 2010.

[4] Naik,P.Daniel, Martin Middendorf, and HartmutSchmeck. "Ant colony optimization for resourceconstrained project scheduling."Evolutionary Computation, IEEE Transactions on 6.4 (2002): 333-346.

[5] Tawfeek M, et al. " Task Scheduling based on Ant Colony Optimization in a distributed cloud." Global Telecommunications Conference (GLOBECOM 2010)

[6] Liu, Jing, et al. "Job scheduling model for cloud computing based on multi-objective genetic algorithm." IJCSI International Journal of Computer Science Issues10.1 (2013): 134-139.

[7] Dillon, Tharam, Chen Wu, and Elizabeth Chang. "Cloud computing: issues and challenges." Advanced Information Networking and Applications (AINA), 2010 24th IEEE International Conference on.Ieee, 2010".

[8] Pandey, Suraj, et al. "A particle swarm optimizationbased heuristic for scheduling workflow applications in cloud computing environments." Advanced Information Networking and Applications (AINA), 2010 24th IEEE International Conference on.IEEE, 2010. 